\title{
ESTUDOS SOBRE A MENSURAÇÃO DA CONDUTIVIDADE ELÉTRICA DO SOLO
}

\author{
JOSÉ P. MOLIN ${ }^{1}$, LADISLAU M. RABELLO ${ }^{2}$
}

RESUMO: A condutividade elétrica é a capacidade que um material possui em conduzir corrente elétrica, e uma das suas utilidades na agricultura provém do fato de que a massa do solo com sua variabilidade na composição físico-química apresenta diferentes níveis de condutividade elétrica (CE). O objetivo do presente trabalho foi estudar o comportamento da CE no solo e avançar no entendimento dos fatores que regem seus níveis de variação e desenvolver sistemas que permitam a mensuração contínua da CE do solo para a geração de mapas. Construiu-se um sistema com várias configurações de mensuração da $\mathrm{CE}$ e, nas avaliações em campo, os resultados foram parcialmente satisfatórios. Num estudo detalhado utilizando apenas um sistema comercial para sua mensuração, obteve-se a indicação clara de que a CE responde às variações na textura do solo e em seus teores de umidade, o que demonstra o potencial que ela tem como ferramenta para facilitar e baratear o processo de obtenção de dados para a caracterização física dos solos.

PALAVRAS-CHAVE: agricultura de precisão, sensores, indicador qualitativo de solo.

\section{STUDIES ABOUT SOIL ELECTRICAL CONDUCTIVITY MEASUREMENTS}

\begin{abstract}
The electric conductivity is the capacity of a material in driving electric current and one of its usefulness in the agriculture comes from the fact that the soil electrical conductivity (EC) varies with its intrinsic physicochemical variability. The objective of this work was to study the EC behavior and advance on the factors understanding that affects its variability, and develops systems for measuring and mapping EC. We built a system with several measurement configurations, and on the field tests the results were partially satisfactory. In a detailed study using only a commercial EC measuring equipment the results clearly indicated that EC relates with soil texture and moisture, and may represent an important and low price tool for collecting data and characterizing soil physical properties.
\end{abstract}

KEYWORDS: precision agriculture, sensors, qualitative soil indicator.

\section{INTRODUÇÃO}

Os elevados níveis de produtividade agrícola estão associados ao uso intenso de insumos. Diante disto, há a necessidade de se desenvolverem sistemas que permitam racionalizar o uso de insumos e, consequentemente, os custos de produção e o impacto ambiental, e ao mesmo tempo incrementar a produção. Essa é a proposta da agricultura de precisão, conceito segundo o qual o gerenciamento da atividade agrícola é feito levando-se em consideração a variabilidade espacial e temporal das culturas e de seus fatores, o que exige, dentre outros, a geração intensificada de dados (ARAUJO et al., 2005; VIEIRA JUNIOR et al., 2006).

A necessidade de caracterização espacial de fatores de planta e de solo tem levado ao surgimento de uma série de propostas e sistemas de sensoriamento e monitoramento; por exemplo, na tentativa de desenvolver técnicas de medida indireta de propriedades do solo, têm sido estudados sistemas ópticos, eletromagnéticos, eletroquímicos, mecânicos, fluxo de ar e acústicos (ADAMCHUK et al., 2004). Dentre estes a condutividade elétrica do solo (CE) tem tido destaque e CORWIN \& LESCH (2005) apresentam uma síntese de aplicações onde ela tem sido utilizada como um indicador no monitoramento de características do solo, como salinidade, textura e

\footnotetext{
${ }^{1}$ Professor Associado, Departamento de Engenharia de Biossistemas, ESALQ/USP, Piracicaba - SP, jpmolin@ usp.br.

${ }^{2}$ Pesquisador A, Embrapa Instrumentação, São Carlos - SP, rabello@ cnpdia.embrapa.br.

Recebido pelo Conselho Editorial em: 28-9-2009

Aprovado pelo Conselho Editorial em: 30-11-2010
} 
estratificação, umidade, densidade, matéria orgânica e derivados, CTC, lixiviação, partição de doses de herbicidas, definição de bordas em classificação de solos, classes de drenagem, recarga de lençol freático, entre outras.

Os primeiros relatos sobre estudos com CE são relacionados à salinidade dos solos, na década de 1970, no "USDA-ARS Salinity Laboratory", em Riverside-CA, EUA (CORWIN \& LESCH, 2005). O teor de água e as propriedades físicas e químicas, em especial a salinidade, podem ser citados como fatores de maior influência, sendo que as mudanças de temperatura também influenciam na CE (RHOADES, 1993).

Para mensurar a CE, geralmente são utilizados o sistema de indução eletromagnética e o de contato direto (RHOADES \& CORWIN,1984). A indução eletromagnética é uma técnica para medir a condutividade elétrica em solos sem contato com o mesmo, sendo um método de amostragem não destrutiva. Um equipamento comercial (EM $38^{\circledR}$, Geonics Limited, Mississauga, Ontario, Canadá), com esse princípio de funcionamento, realiza uma medida de condutividade elétrica integrada até a profundidade em torno de $1,5 \mathrm{~m}$ na posição vertical e a uma profundidade de aproximadamente 0,3 m na posição horizontal (MOLIN et al., 2005). O sistema por contato direto penetra no solo, geralmente utilizando como eletrodos de fluxo de corrente elétrica, discos de corte lisos, e capta a condutividade elétrica a uma profundidade diretamente proporcional aos espaçamentos entre os eletrodos. Um equipamento que utiliza esse princípio de funcionamento (Veris ${ }^{\circledR}$, Veris Thecnologies, Salina, KS, EUA) fornece valores de condutividade elétrica sem nenhuma calibração (MACHADO et al., 2006). Pesquisa realizada por SUDDUTH et al. (2005), comparando as duas tecnologias, concluiu que ambos os equipamentos fornecem dados similares. Além destes, estão disponíveis outros sistemas na investigação da distribuição espacial da CE e de parâmetros a ela relacionados, inclusive no auxílio a decisões na aplicação localizada de insumos (LÜCK et al., 2009).

As principais linhas de estudo referentes aos fatores que influenciam na CE estão concentradas em propriedades como salinidade, teor de água, textura e algumas propriedades químicas de interesse agrícola, como a CTC. Isso é devido a uma boa correlação existente na distribuição destes fatores no solo com o mapeamento da condutividade elétrica (RHOADES, 1993; MOLIN et al., 2005; MACHADO et al., 2006; MOLIN \& CASTRO, 2008). Em um estudo em escala que contemplou vários estados do meio-oeste americano, SUDDUTH et al. (2005) avaliaram as relações entre a CE medida por indução e por contato, e suas relações com fatores do solo, observando boas correlações entre as duas formas de medição e da CE com textura e CTC dos solos.

Uma abordagem inovadora na alocação de parcelas experimentais é apresentada por JOHNSON et al. (2005) e que permite a redução de erro experimental, tanto em parcelas como em experimentação em escala de campo, com base numa classificação da variância da $\mathrm{CE}$ da área experimental. A partir desse mesmo princípio, a possibilidade de gerar o mapeamento da $\mathrm{CE}$ tornou-se uma ferramenta eficiente na investigação do comportamento e da variabilidade espacial de propriedades do solo, pois permite identificar áreas com propriedades semelhantes e delimitar unidades de gerenciamento (FRAISSE et al., 2001; MOLIN \& CASTRO, 2008).

Como o tema ainda carece de maior domínio, em especial em nosso meio, este trabalho objetivou o entendimento e o estudo do comportamento da CE de solos, locais utilizando parcelas com texturas distintas e regressões da CE com o teor de argila e teor de água no solo em diferentes mensurações ao longo do tempo, bem como o desenvolvimento de um sistema de mensuração da CE para aplicações agrícolas.

\section{MATERIAL E MÉTODOS}

Inicialmente, trabalhou-se na proposta de um sistema de mensuração e mapeamento da $\mathrm{CE}$ que poderia ser associado à operação de semeadura direta, em função de as semeadoras utilizarem 
em sua estrutura básica componentes que facilitam o processo, além de permitir o levantamento dos dados na operação de semeadura.

Foram propostos dois sistemas envolvendo duas metodologias de mensuração da CE: o sistema Resistência Elétrica do Solo (RE) e o sistema Duas Pontas (DP), ambos utilizando discos de corte lisos de 0,40 m de diâmetro como eletrodos que penetram no solo, dispostos linearmente em uma barra porta-ferramenta. O princípio de funcionamento desses sistemas é descrito por RUNYAN (1975), onde a condutividade elétrica é obtida a partir da resistividade elétrica de um meio. Assim, tem-se:

$$
\sigma=\frac{1}{\rho}
$$

em que,

$$
\begin{aligned}
& \sigma \text { - condutividade elétrica, } \mathrm{S} \mathrm{m}^{-1} \text {, e } \\
& \rho \text { - resistividade elétrica, } \Omega \mathrm{m} \text {. }
\end{aligned}
$$

O sistema RE é uma adaptação dinâmica da obtenção da resistividade elétrica de um material, utilizando uma amostra retangular de dimensões conhecidas para medir a sua resistência elétrica com dois eletrodos, conforme a relação:

$$
\rho=R \frac{A}{L}
$$

em que,

$\mathrm{R}$ - resistência elétrica, $\Omega$;

L - comprimento da amostra, m, e

A - área da seção transversal, $\mathrm{m}^{2}$.

O sistema DP segue o princípio dos dois eletrodos ou duas pontas, e sua configuração consiste em utilizar dois eletrodos afastados por uma distância (S) e aplicar uma corrente elétrica (I) a fim de medir a alteração no potencial elétrico (V), na região em que a corrente elétrica transitou. Este método é definido pela equação:

$$
\rho=\frac{\mathrm{A}}{\mathrm{I}} \frac{\mathrm{dV}}{\mathrm{dx}}
$$

em que,

I - corrente elétrica, A;

A - área da seção transversal, $\mathrm{m}^{2}$, e

$\frac{\mathrm{dV}}{\mathrm{dX}}$ - relação entre a leitura de tensão e a distância entre os eletrodos.

A fim de respeitar os princípios de funcionamento de cada sistema e simular a estrutura de semeadoras para semeadura direta, em que os discos de corte podem servir como eletrodos, uma fonte de tensão estabilizada foi projetada e construída.

Ela serviu para a alimentação dos sistemas com a finalidade de baixar e estabilizar a tensão elétrica de $12 \mathrm{Vdc}$, adquirida de uma bateria, para o funcionamento do sistema em $5 \mathrm{Vdc}$, a fim de evitar qualquer oscilação na tensão da bateria, provocada com o consumo de sua carga. Também foi construído um circuito paralelo ao estabilizador de tensão, com capacitores, reguladores e resistências elétricas (Figura 1). 


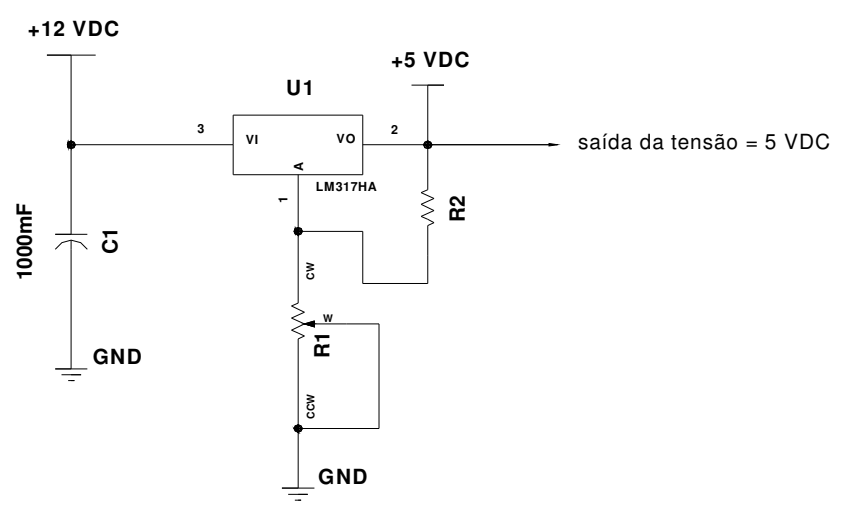

(a)

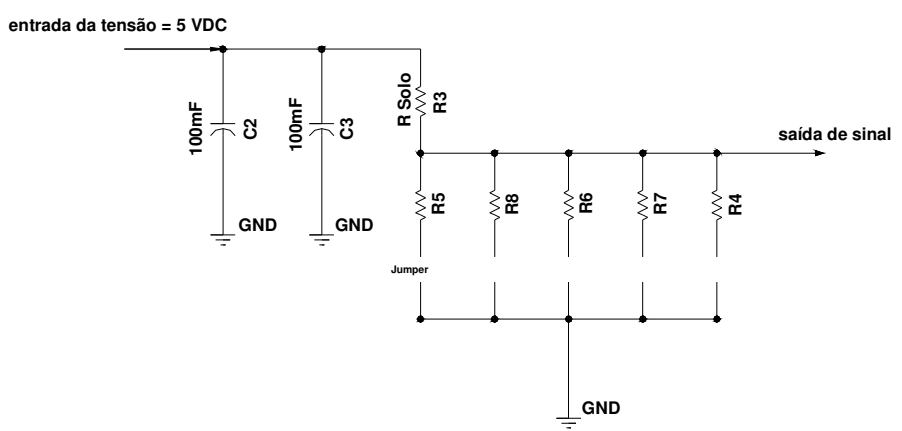

(b)

FIGURA 1. Esquema do circuito regulador de tensão para alimentação dos sistemas (a) e do circuito de mensuração da resistência elétrica do solo (b). Schematic representation of the tension circuit for systems input (a) and soil electrical resistance measurement circuit (b).

A aquisição de dados foi feita utilizando-se de um coletor de dados desenvolvido especificamente para este fim. $O$ coletor teve a função de captar as medidas analógicas de resistência elétrica do solo, georreferenciadas por um receptor de GPS, e enviá-las no formato digital para um computador de mão. As medidas de resistência elétrica eram convertidas em condutividade elétrica e armazenadas em formato de texto por meio de um programa computacional desenvolvido especialmente para essa aplicação.

Construiu-se uma estrutura mecânica (Figura 2) que englobou a captação de dados de CE nos dois sistemas desenvolvidos, composta de dois discos de corte lisos, de 0,4 m de diâmetro, exercendo a função de eletrodos, que penetram no solo, dispostos linearmente em uma barra portaferramenta.

Para o sistema RE, foram utilizadas rodas de borracha limitadoras de penetração, acopladas de forma concêntrica aos discos de corte, permitindo a máxima penetração de $0,10 \mathrm{~m}$. Considerou-se a geometria de um bloco de solo entre os eletrodos, definido pela distância entre estes e a área do setor semicircular do disco de corte dentro do solo.

Para a realização do levantamento de dados com o sistema DP, não foi necessária nenhuma limitação quanto à penetração dos discos de corte ao solo, mas somente a garantia de contato do disco com o solo. Os discos foram isolados da barra porta-ferramenta por placas de PVC, a partir de seus suportes. 


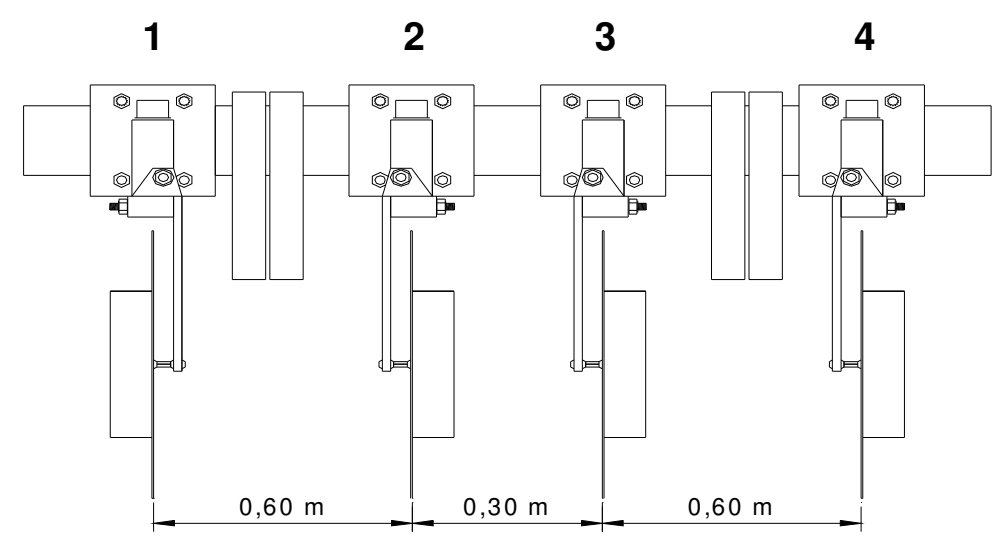

Barra porta-

-ferramenta

Discos

FIGURA 2. Desenho esquemático da estrutura mecânica composta pela barra porta-ferramenta e os quatro discos de corte. Schematic drawing of the mechanical device composed by the attaching bar and four cutting disks.

Na configuração da estrutura mecânica, foi possível compor com o sistema RE variando os espaçamentos entre os discos e com o sistema DP fazendo a troca de resistências elétricas (R4, R5, R6, R7 e R8, Figura 1) no circuito de mensuração, por meio de um "jumper". O circuito foi construído com as cinco resistências elétricas ligadas em paralelo para permitir verificar a melhor sensibilidade de leitura na $\mathrm{CE}$.

Para isso, foi estabelecida a utilização das resistências elétricas R8, R6 e R7, respectivamente de 27; 47 e $220 \Omega$ e apenas um espaçamento de 0,6 m entre os discos. Assim, com esse sistema, foi possível compor três sistemas: resistência elétrica de $27 \Omega$ e espaçamento de $0,6 \mathrm{~m}$ (DP 27); resistência elétrica de $47 \Omega$ e espaçamento de $0,6 \mathrm{~m}$ (DP 47), e resistência elétrica de $220 \mathrm{ohm}$ e espaçamento de 0,6 m (DP 220). Esse espaçamento foi escolhido para possibilitar a medida da condutividade elétrica do solo através das linhas de campo elétrico descrito pela semicircunferência da distância entre os dois eletrodos, ocasionando uma coleta de condutividade na profundidade de 0 a $0,3 \mathrm{~m}$.

As distâncias entre os discos de corte foram estabelecidas para que fosse possível mensurar a resistência elétrica do solo, alternando o espaçamento entre eletrodos em três possíveis combinações com o sistema RE. Dessa forma, eram produzidos blocos de solo com o comprimento de $0,3 \mathrm{~m}$ entre o segundo e o terceiro, $0,6 \mathrm{~m}$ entre o primeiro e o segundo e $0,9 \mathrm{~m}$ entre o segundo e o quarto disco; neste caso, com a retirada do terceiro disco.

Para testar os dispositivos, foi executado um experimento de campo em uma área pertencente ao Câmpus da USP/ESALQ, em Piracicaba - SP. O equipamento utilizado como referência na coleta de dados de CE foi o sistema comercial Veris 3100, que permite a mensuração de dados nas profundidades de 0 a $0,3 \mathrm{~m}$ e de 0 a $0,9 \mathrm{~m}$ a partir do contato direto de discos de corte lisos, sendo um par para eletrodos de fluxo de corrente e um par para a medida de tensão elétrica.

Com o auxílio desse equipamento, foram alocadas cinco áreas, de $25 \mathrm{~m}^{2}$ cada uma $(2,5 \mathrm{~m}$ por $10,0 \mathrm{~m}$ ), e delimitadas no local de tal forma que apresentassem grande variação textural entre elas. O solo das áreas variou de Argissolo Vermelho-Amarelo textura média/argilosa para textura arenosa/média.

As mensurações de condutividade elétrica e umidade do solo foram efetuadas em três épocas distintas (abril, maio e agosto de 2006). Em cada parcela, foram testadas as seis combinações dos sistemas propostos, tendo sido escolhida apenas uma resistência elétrica de trabalho (R8) no mesmo circuito já descrito.

Além disso, também foi utilizado o sistema referência para avaliar o efeito do teor de umidade no solo. Foram feitas três leituras em datas que ofereciam condições distintas de umidade do solo. 
Ao final, analisaram-se os valores de CE e suas possíveis relações com os valores de granulometria e teores de umidade do solo.

A textura do solo foi obtida a partir de uma amostra compostas de três subamostras retiradas com um trado de rosca na profundidade de 0 a $0,30 \mathrm{~m} ; 0,30$ a $0,60 \mathrm{~m}$, e 0,60 a $0,90 \mathrm{~m}$ em cada parcela e utilizando na sua determinação o método de Bouyoucos.

As observações nas três camadas visaram a avaliar possíveis disparidades significativas na composição textural do perfil das parcelas, considerando que cada combinação dos sistemas deve executar leituras dentro de uma determinada espessura de perfil. $\mathrm{O}$ teor de umidade foi obtido a partir de amostras coletadas da mesma forma, porém repetidas para cada leitura dos valores de CE, utilizando o método gravimétrico (EMBRAPA, 1999).

Um segundo experimento foi conduzido em uma área próxima ao primeiro, visando a explorar com mais ênfase o sistema referência e buscar o maior entendimento do comportamento da $\mathrm{CE}$ no solo, especialmente fazendo variar o teor de umidade e a granulometria do solo. Para a coleta dos dados, foram delimitadas seis áreas, de $30 \mathrm{~m}^{2}$ cada uma ( $3 \mathrm{~m}$ por $10 \mathrm{~m}$ ), e delimitadas no local de tal forma que apresentassem grande variação textural entre elas, e as mensurações de condutividade elétrica e a umidade do solo foram efetuadas em três datas distintas (4-10; 10-11 e 7-12-2006), visando a obter condições distintas de umidade do solo.

A CE foi medida em onze pontos espaçados de modo semelhante dentro de cada uma das seis parcelas, nas três visitas efetuadas. Os valores de CE foram obtidos com o equipamento parado durante a mensuração para cada ponto, sendo lidos e anotados pelo operador. Os teores de umidade e a textura foram obtidos seguindo o procedimento anterior.

A partir dos dados de CE, textura e teores de umidade do solo, foram conduzidas análises de regressão polinomial, utilizando o programa computacional SAS (1998) com o teste F de Snedecor, possibilitando a escolha entre o melhor modelo, sendo escolhidos os modelos, significativos, com maior coeficiente de determinação.

\section{RESULTADOS E DISCUSSÃO}

Os conteúdos de argila e areia das cinco parcelas selecionadas para testar os sistemas propostos (Tabela 1) demonstram variação textural entre as parcelas, embora algumas tenham apresentado valores semelhantes entre si, especialmente as parcelas 2; 3 e 4. Já entre as profundidades, as diferenças são pouco expressivas, apenas indicando maiores teores de areia na camada mais superficial, especialmente nas parcelas 3 e 5 .

Os resultados foram obtidos com os sistemas propostos para a mensuração da CE (Figura 3). A análise do gráfico referente à primeira leitura mostra que as mensurações do sistema de referência apresentaram valores de CE crescentes, na medida em que os teores de argila das parcelas se tornavam maiores, o que é o padrão esperado, já que o teor de argila é um dos fatores determinantes na capacidade do solo de conduzir corrente elétrica.

Nas demais leituras, no entanto, essa tendência não foi observada. Considerando-se que a condição de umidade do solo baixou entre as leituras, em função do período de secas natural da região, o baixo teor de água no solo deve ter influenciado as leituras.

Ainda, na primeira leitura, as combinações DP 220, DP 47, RES 0,6 e RES 0,9 apresentaram os maiores valores de CE na parcela 3. A configuração RES 0,9 foi a que resultou nos maiores valores de CE, enquanto o DP 27 apresentou os menores valores. 
TABELA 1. Teores de argila e areia no solo das cinco parcelas selecionadas para o experimento com os sistemas propostos. Clay and sand content on the soil of the five selected plots for the experiment with the proposed systems.

\begin{tabular}{cccc}
\hline Parcela & Profundidade $(\mathrm{cm})$ & Argila $\left(\mathrm{g} \mathrm{kg}^{-1}\right)$ & Areia \\
\hline \multirow{2}{*}{1} & $0-30$ & 102,5 & 806,6 \\
& $30-60$ & 107,4 & 814,5 \\
& $60-90$ & 115,4 & 782,7 \\
\hline \multirow{2}{*}{2} & $0-30$ & 308,2 & 560,1 \\
& $30-60$ & 371,6 & 520,1 \\
\multirow{2}{*}{3} & $60-90$ & 328,8 & 530,1 \\
\hline & $0-30$ & 228,7 & 560,8 \\
\multirow{2}{*}{4} & $30-60$ & 347,9 & 494,0 \\
& $60-90$ & 360,6 & 472,5 \\
\hline \multirow{2}{*}{5} & $0-30$ & 136,4 & 489,7 \\
& $30-60$ & 370,7 & 462,6 \\
& $60-90$ & 379,4 & 444,5 \\
\hline
\end{tabular}

A segunda leitura foi realizada em condições de teor de umidade mais baixa no solo e novamente os valores de CE fornecidos pelo sistema referência, nas duas profundidades, apresentaram valores crescentes, na medida em que os teores de argila do solo aumentavam. Nos resultados dos sistemas sob avaliação, verificou-se um mesmo padrão, acentuado, sobretudo na configuração RES 0,9, RES 0,6 e RES 0,3. Nesta etapa, por problemas na instrumentação, a configuração DP 47 não pôde ser avaliada.

A terceira leitura foi realizada em condições bastante secas, que são inadequadas à mensuração da $\mathrm{CE}$, já que a água é o principal meio condutor da corrente. Dessa maneira, as configurações DP 27 e DP 47 não apresentaram mensurações em nenhuma parcela. Na parcela 1, somente o sistema de referência realizou alguma mensuração, em ambas as profundidades, e apresentou o mesmo padrão das leituras anteriores. Os sistemas RES 0,9 e RES 0,6 apresentaram os maiores valores na parcela 3 e tendência de crescimento, na medida em que aumentava o teor de argila das parcelas.

Um problema verificado em relação ao método Resistência Elétrica do Solo foi a profundidade do disco de corte, que deveria ser mantida em $0,10 \mathrm{~m}$. No entanto, em solo úmido, este aderia à roda de controle de profundidade e, em solo muito seco, os discos não aprofundavam o necessário. O sistema referência e o de Duas Pontas não apresentam esse problema, pois nestes basta o contato do disco com o solo para a mensuração. Por essa razão e pelos resultados, que embora não plenamente consistentes, é indicado que a mensuração pelo método das pontas, ou seja, medindo a condutividade diretamente e não a resistência do solo, é mais efetivo e exequível em campo. Alguns aspectos ainda deverão ser explorados nesse circuito para que as leituras sejam, por exemplo, equivalentes aos valores obtidos com o sistema referência. Em primeira instância, o sistema deve ser sensível às variações nos teores de argila do solo, como já observado em solos brasileiros (MACHADO et al., 2006; MOLIN \& CASTRO, 2008).

Os conteúdos de argila, silte e areia das seis parcelas utilizadas no estudo (Tabela 2) demonstram variação textural entre as parcelas, o que permitiu o contraste desejado para as mensurações de CE e de suas relações com os demais fatores. O desejado seria que essa variação fosse ainda maior, indicando que a condição ideal de trabalho seria a montagem de condições de solo artificiais em grandes caixas ou canais de solo. No entanto, para os propósitos desta etapa da investigação, foi possível observar que os valores de CE média variaram em cada parcela, em 
função da época de coleta dos dados (Figura 4). Esta variação pode ser atribuída à variação no teor de água do solo, visto que, em curto espaço de tempo, a textura do solo não varia e pode ser considerada como constante. Já a variação dos dados de CE, quando comparadas parcelas distintas na mesma época (Figura 6), pode ser atribuída aos diferentes conteúdos de argila em cada área, destacando a propriedade da argila de reter mais água e também de conduzir mais eletricidade que areia ou silte.

\section{Experimento 1}

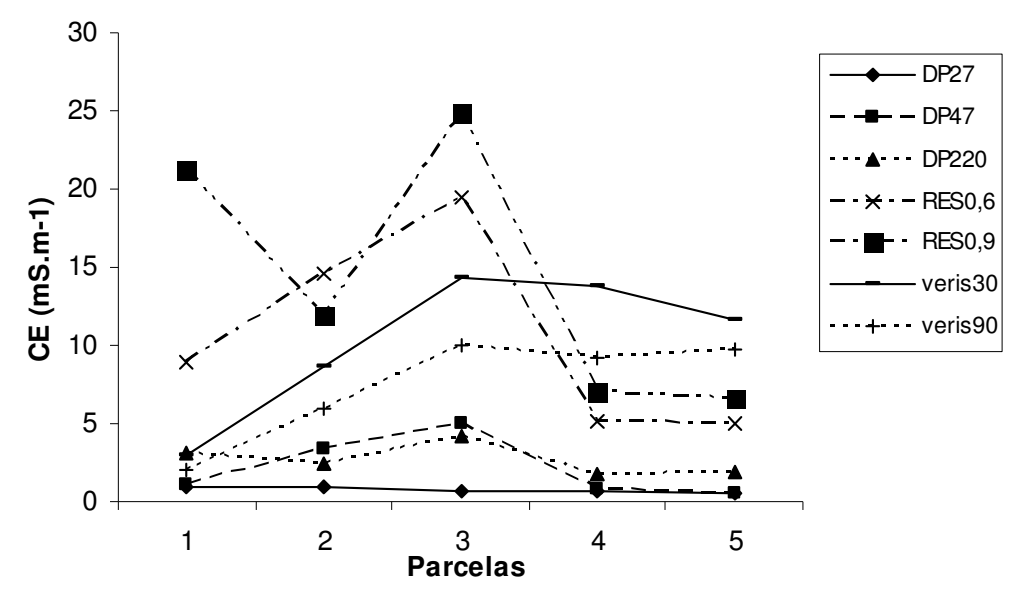

Experimento 2

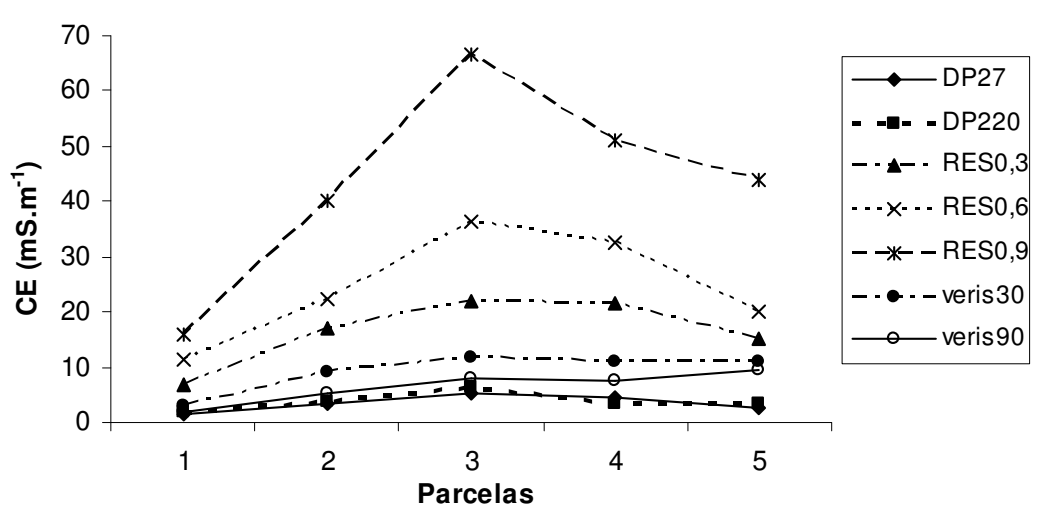

Experimento 3

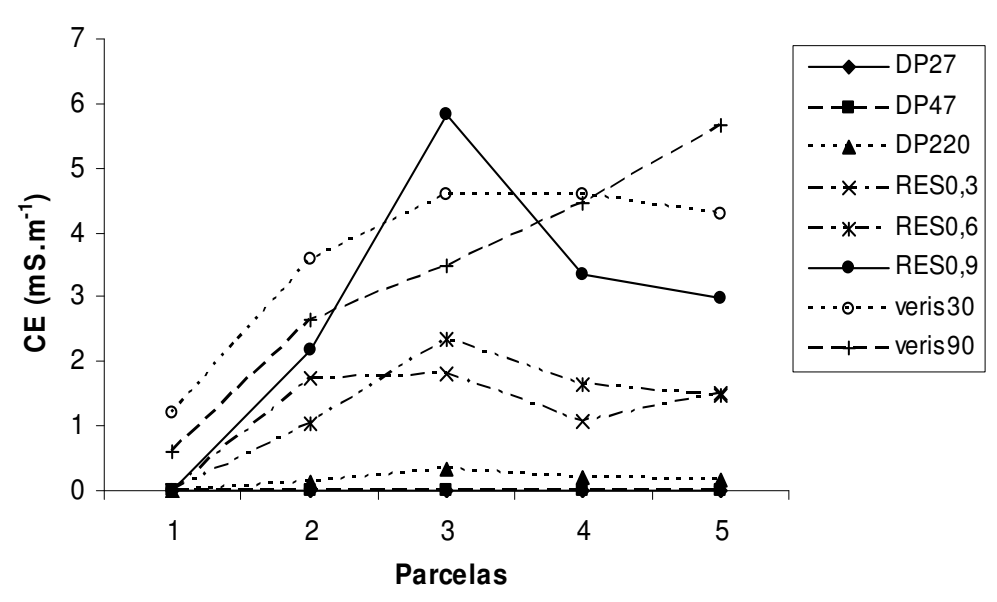

FIGURA 3. Gráficos dos valores de CE obtidos com os sistemas avaliados, em função das parcelas experimentais. Graphics of EC values obtained with the evaluated systems, as function of the experimental plots. 
TABELA 2. Teores de argila, silte e areia nas seis parcelas selecionadas para o segundo experimento. Clay, silt and sand contents on the six plots selected for the second experiment.

\begin{tabular}{cccc}
\hline Parcela & Argila & Silte $\left(\mathrm{g} \mathrm{kg}^{-1}\right)$ & Areia \\
\hline 1 & 370,0 & 140,9 & 489,1 \\
2 & 302,5 & 153,4 & 544,1 \\
3 & 239,0 & 190,8 & 570,2 \\
4 & 268,4 & 123,4 & 608,2 \\
5 & 110,6 & 142,0 & 747,4 \\
6 & 91,9 & 73,4 & 834,7 \\
\hline
\end{tabular}

Na análise de regressão entre a condutividade elétrica e o conteúdo de argila do solo (Figura 4), obtiveram-se equações polinomiais de primeiro grau com elevados valores de coeficientes de determinação $\left(\mathrm{R}^{2}\right)$, indicando que, quanto maior o conteúdo de argila apresentado pelo solo, na área de estudo, maiores foram os valores de condutividade elétrica.

Já a análise de regressão entre a CE e a umidade do solo (Figura 5) foi representada por uma equação polinomial de segundo grau, indicando que, quanto maior o teor de umidade, medida na área de estudo, maiores foram os valores de CE encontrados na área. Foi realizada também a análise de regressão entre o teor de umidade e o conteúdo de argila do solo (Figura 6), representada por equações polinomiais de primeiro grau, indicando que o conteúdo de argila do solo afeta positivamente o conteúdo de água armazenado.

Os dados demonstram grande variação nos valores obtidos de CE média do solo quando comparadas áreas diferentes em mesmas épocas e a mesma área em ocasiões deferentes, o que pode ser explicado pela relação da CE com os teores de água e argila do solo (MACHADO et al., 2006).

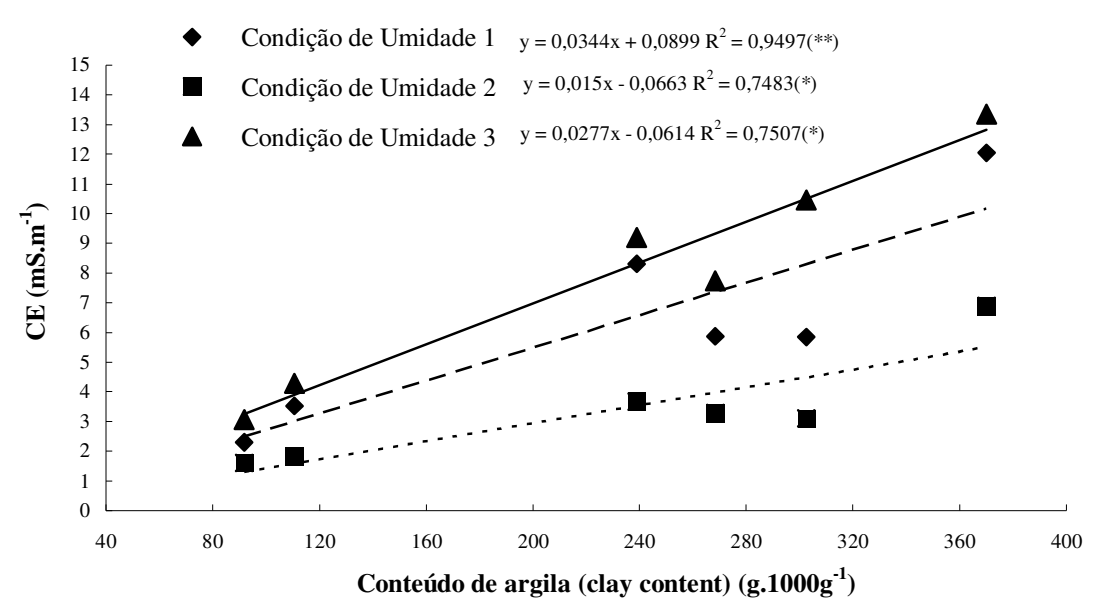

(*) Valor estatisticamente significativo, pelo teste F, com nível de significância de 5\%. (**) Valor estatisticamente significativo, pelo teste F, com nível de significância de $1 \%$.

FIGURA 4. Análise de regressão entre a CE e o conteúdo de argila do solo para as três mensurações equivalentes a teores crescentes de umidade no solo. Regression analysis between EC and clay content for the three measurements equivalent to increasing soil moisture. 


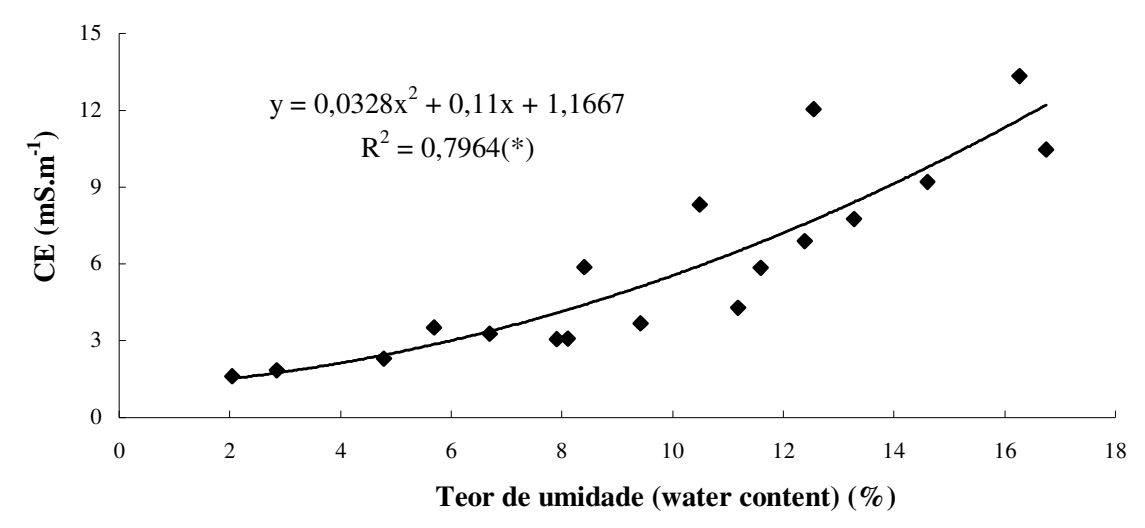

(*) Valor estatisticamente significativo, pelo teste F, com nível de significância de 5\%.

FIGURA 5. Análise de regressão entre a condutividade elétrica e o teor de umidade do solo para todas as mensurações equivalentes a teores crescentes de umidade no solo. Regression analysis of soil electrical conductivity and soil moisture for all measurements equivalent to crescent soil moisture levels.

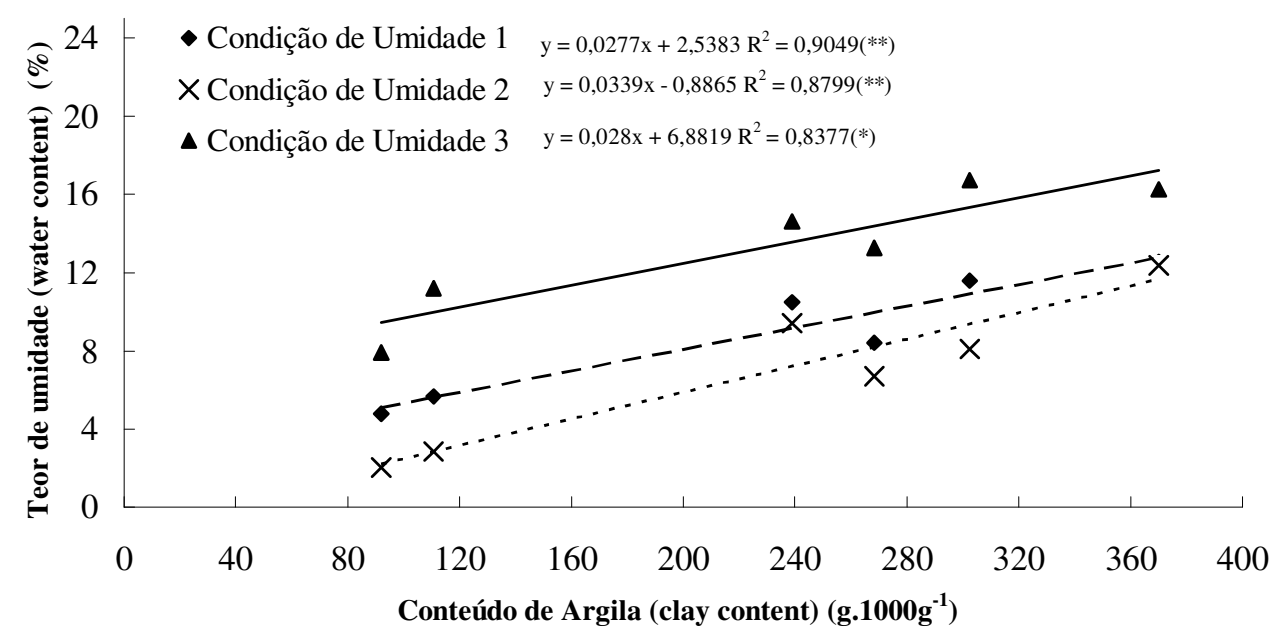

(*) Valor estatisticamente significativo, pelo teste F, com nível de significância de 5\%. (**) Valor estatisticamente significativo, pelo teste F, com nível de significância de $1 \%$

FIGURA 6. Análise de regressão entre os teores de umidade e o conteúdo de argila do solo para as três mensurações equivalentes a teores crescentes de umidade no solo. Regression analysis between soil moisture and clay content for the three measurements equivalent to increasing soil moisture.

Pelos valores elevados dos coeficientes de determinação $\left(R^{2}\right)$, é possível confirmar a dependência entre os fatores estudados, demonstrando que houve interação principalmente entre a $\mathrm{CE}$ e a textura do solo. As correlações entre teores de umidade e CE também foram elevadas, o que demonstra o grande potencial que a medição da condutividade elétrica do solo tem como ferramenta para facilitar e baratear o processo de obtenção de dados, especialmente para a caracterização física dos solos. No entanto, uma limitação permanece, considerando-se que a capacidade de retenção de água do solo é fortemente dependente da sua textura. A mensuração da CE permite a identificação da combinação de ambos os fatores, indicando que valores elevados de CE contrastando com valores menores em outras porções, dentro de uma mesma área, podem estar identificando que os teores de argila, neste caso, são maiores, porém o teor de água é quem está magnificando esse resultado. Enfatiza-se, assim, a necessidade da confirmação com a mensuração ou estimação do teor de água. 


\section{CONCLUSÕES}

Os estudos realizados utilizando um equipamento comercial mostraram que a CE responde às variações na textura do solo e nos seus teores de umidade, indicando que ela tem potencial para ser utilizada na obtenção de dados para a caracterização física dos solos como uma solução de baixo custo. Já os resultados obtidos com o sistema proposto não foram plenamente satisfatórios porque não foi possível obter uma resposta consistente na CE às variações de teores de argila e de umidade no solo, indicando que o sistema deve ainda evoluir.

\section{AGRADECIMENTOS}

Ao CNPq e à FAPESP, pelo apoio financeiro ao projeto, e aos estudantes André Pincelli, Rodrigo Portugal Sousa, Mark Spekken e Marston Héracles Domingues Franceschini, pelo apoio nas atividades de campo.

\section{REFERÊNCIAS}

ADAMCHUK, V.I.; HUMMEL, J.W.; MORGAN, M.T.; UPADHYAYA, S.K. On-the go soil sensors for precision agriculture. Computers and Electronics in Agriculture. Amsterdam, v.44, p.71-91, 2004.

ARAUJO, J.C.; VETTORAZZI, C.A.; MOLIN, J.P. Estimativa da produtividade e determinação de zonas de manejo, em cultura de grãos, por meio de videografia aérea multiespectral. Acta Scientiarum, Maringá, v.27, n.3, p.437-447, 2005.

CORWIN, D.L.; LESCH, S.M. Apparent soil electrical conductivity measurements in agriculture. Computers and Electronics in Agriculture, Amsterdam, v.46, p.11-43, 2005.

EMBRAPA. EMPRESA BRASILEIRA DE PESQUISA AGROPECUÁRIA. Sistema brasileiro de classificação de solos. Brasília: Embrapa Produção de Informação, 1999.

FRAISSE, C.W.; SUDDUTH, K.A.; KITCHEN, N.R. Delineation of site-specific management zones by unsupervised classification of topographic attributes and soil electrical conductivity. Transations ASAE, St. Joseph, v.44, p.155-166, 2001.

JOHNSON, C.K.; ESKRIDGE, K.M.; CORWIN, D.L. Apparent soil electrical conductivity: applications for designing and evaluating field-scale experiments. Computers and Electronics in Agriculture, Amsterdam, v.46, p.181-202, 2005.

LÜCK, E.; SPANGENBERG, U.; RUEHLMANN, J. Comparison of different EC-maping sensors. In: HENTEN, E.J.; GOENSE, D.; LOKHORST, C. (Ed.). Precision Agriculture '09. Wageningen: Academic Publishers, 2009. p.445-452.

MACHADO, P.L.O.A.; BERNARDI. A.C.C.; VALENCIA, L.I.O.; MOLIN, J.P.; GIMENEZ, L.; SILVA, C.A.; ANDRADE, A.G.;MADARI, B.E.; MEIRELLES, M.S.P. Mapeamento da condutividade elétrica do solo e relação com os teores de argila de um Latossolo Vermelho sob plantio direto no Paraná. Pesquisa Agropecuária Brasileira. Brasília, v.41, n.6, p.1.023-1.031, 2006.

MOLIN, J.P.; GIMENEZ, L.M.; PAULETTI, V.; SCHMIDHALTER, U.; HAMMER, J. Mensuração da condutividade elétrica do solo por indução e sua correlação com fatores de produção. Engenharia Agrícola, Jaboticabal, v.25, n.2, p.420-426, 2005.

MOLIN, J.P.; CASTRO, C.N. Establishing management zones using soil electrical conductivity and other soil properties by the fuzzy clustering technique. Scientia Agricola, Piracicaba, v.65, p.567$573,2008$.

RHOADES, J.D.; CORWIN, D.L. Measurement of inverted electrical conductivity profiles using electromagnetic induction. Soil Science Society of America Journal, Madison, v.44, p.288-291, 1984. 
RHOADES, J. D. Electrical conductivity methods for measuring and mapping soil salinity. Advances in Agronomy, San Diego, v.49, p.232-242, 1993.

RUNYAN, W.R. Semiconductor measurements and instrumentation: resistivity and carrierconcentration measurements. New York: McGraw-Hill, 1975. 280 p.

SAS Institute. SAS/SAT: user's guide: Version 6. Cary, 1998. 1.848 p.

SUDDUTH, K.A.; KITCHEN, N.R.; WIEBOLD, W.J.; BATCHELOR, W.D.; BOLLERO, G.A.; BULLOCK, D.G.; CLAY, D.E.; PALM, H.L.; PIERCEF, F.J.; SCHULER, R.T.; THELEN, K.D. Relating apparent electrical conductivity to soil properties across the north-central USA. Computers and Electronics in Agriculture, New York, v.46, p.263-283, 2005.

VIEIRA JÚNIOR, P.A.; MOLIN, J.P.; DOURADO NETO, D.; MANFRON, P.A.; MASCARIN, L.S.; FAULIN, G.C.; DETOMINI, E.R. População de plantas e alguns atributos do solo relacionados ao rendimento de grãos de milho. Acta Scientiarum Agronomy, Maringá, v.28, p.483492, 2006. 